\title{
BMJ Open Chinese patients with hereditary spastic paraplegias (HSPs): a protocol for a hospital-based cohort study
}

Yu-Sen Qiu, ${ }^{1}$ Yi-Heng Zeng, ${ }^{1}$ Ru-Ying Yuan, ${ }^{1}$ Zhi-Xian Ye, ${ }^{1}$ Jin Bi, ${ }^{1}$ Xiao-Hong Lin, ${ }^{1}$ Yi-Jun Chen, ${ }^{1}$ Meng-Wen Wang, ${ }_{1}^{1}$ Ying Liu, ${ }^{2,3}$ Shao-Bo Yao, ${ }^{4}$ Yi-Kun Chen, ${ }^{1}$ Jun-Yi Jiang, ${ }^{1}$ Yi Lin, ${ }^{1}$ Xiang Lin, ${ }^{1}$ Ning Wang, ${ }^{1}$ Ying Fu (D) , ${ }^{1}$ Wan-Jin Chen (D) ${ }^{1}$
To cite: Qiu Y-S, Zeng Y-H, Yuan R-Y, et al. Chinese patients with hereditary spastic paraplegias (HSPs): a protocol for a hospital-based cohort study. BMJ Open 2022;12:e054011. doi:10.1136/ bmjopen-2021-054011

- Prepublication history and additional supplemental material for this paper are available online. To view these files, please visit the journal online (http://dx.doi.org/10.1136/ bmjopen-2021-054011)

Y-SQ, Y-HZ and R-YY contributed equally.

Received 02 June 2021 Accepted 25 November 2021

Check for updates

(c) Author(s) (or their employer(s)) 2022. Re-use permitted under CC BY-NC. No commercial re-use. See rights and permissions. Published by BMJ.

For numbered affiliations see end of article.

Correspondence to

Dr Wan-Jin Chen;

wanjinchen75@fjmu.edu.cn

\section{ABSTRACT}

Introduction Hereditary spastic paraplegias (HSPs) are uncommon but not rare neurodegenerative diseases. More than 100 pathogenic genes and loci related to spastic paraplegia symptoms have been reported. HSPs have the same core clinical features, including progressive spasticity in the lower limbs, though HSPs are heterogeneous (eg, clinical signs, MRI features, gene mutation). The age of onset varies greatly, from infant to adulthood. In addition, the slow and variable rates of disease progression in patients with HSP represent a substantial challenge for informative assessment of therapeutic efficacy. To address this, we are undertaking a prospective cohort study to investigate genetic-clinical characteristics, find surrogates for monitoring disease progress and identify clinical readouts for treatment. Methods and analysis In this case-control cohort study, we will enrol 200 patients with HSP and 200 healthy individuals in parallel. Participants will be continuously assessed for 3 years at 12-month intervals. Six aspects, including clinical signs, genetic spectrum, cognitive competence, MRI features, potential biochemical indicators and nerve electrophysiological factors, will be assessed in detail. This study will observe clinical manifestations and disease severity based on different molecular mechanisms, including oxidative stress, cholesterol metabolism and microtubule dynamics, all of which have been proposed as potential treatment targets or modalities. The analysis will also assess disease progression in different types of HSPs and cellular pathways with a longitudinal study using $\mathrm{t}$ tests and $\chi^{2}$ tests.

Ethics and dissemination The study was granted ethics committee approval by the first affiliated hospital of Fujian Medical University (MRCTA, ECFAH of FMU (2019)194) in 2019. Findings will be disseminated via presentations and peer-reviewed publications. Dissemination will target different audiences, including national stakeholders, researchers from different disciplines and the general public.

Trial registration number NCT04006418.

\section{INTRODUCTION}

Hereditary spastic paraplegias (HSPs) are genetically driven disorders characterised by progressive spastic gait disturbance. Lengthdependent corticospinal tracts and dorsal

\section{Strengths and limitations of this study}

- This is a first case-control, parallel cohort study designed to identify surrogates for monitoring disease progress.

- This study will observe disease severity based on different molecular mechanisms that have been proposed as potential treatment targets.

- This is a large longitudinal study with comprehensive data collection.

- Selection bias is inevitable due to the discontinuous enrolment of this study.

column degeneration are hallmark traits of HSPs that lead to the core clinical features of bilateral lower limb spasticity, hyperreflexia and extensor plantar responses. ${ }^{1}$ HSPs are uncommon but not rare, with a combined prevalence of 2-5 per 100000 individuals worldwide. $^{2}{ }^{3}$ The genetic patterns of this family of diseases have been characterised and include autosomal dominant inheritance, autosomal recessive inheritance, X-linked inheritance and mitochondrial inheritance. ${ }^{4}$ To date, more than 100 pathogenic genes and loci related to spastic paraplegia symptoms have been reported, ${ }^{5}$ of which 83 have been formally named and listed as spg1-83, according to their sequence of discovery. ${ }^{6}$

HSPs are generally divided into pure and complicated forms, according to the Anita Harding's classification. ${ }^{78}$ 'Pure' HSP is characterised by isolated pyramidal syndrome, but this may also have co-occurrence with neurogenic bladder disturbances and impairment of vibration sense. 'Complicated' forms of HSP are associated with additional system involvement (cognitive impairment, ataxia, basal ganglia symptoms, visual or auditory disturbances, symptoms of peripheral nerve involvement) and are generally consistent with a more severe disease course. ${ }^{89}$ 
Any HSP is a progressive disease. As the disease progresses, the patient may require crutches or wheelchair assistance. A previous cohort study has shown that the median disease duration until loss of independent walking ability was 22 years. The median time from using a walking aid to the use a wheelchair was 16 years. ${ }^{10}$

Nearly half of patients with HSP have not yet received a genetic diagnosis. ${ }^{10}$ The common clinical and pathological features of different HSPs prefigure certain common themes at the cellular level, and the genetic heterogeneity provides a significant advantage in identifying these convergent themes. ${ }^{11}$ Indeed, published studies have indicated that HSP disease proteins cluster within a wide spectrum of predicted cellular processes: neuronal development, myelin formation and maintenance, microtubule transport, cholesterol metabolism, amino acid metabolism, endosomal and vesicle dynamics, endoplasmic reticulum dynamics, mitochondrial function, purine metabolism and autophagy regulation are the common pathogenetic themes in the HSPs. ${ }^{12}$ Understanding the mechanistic contributions of HSP subtypes to disease variety and severity will allow for data set integration and an attempt to employ innovative means of classifying HSPs according to their molecular mechanisms.

Currently, there is no specific treatment for any form of HSP. ${ }^{2}$ The development of improved treatment methods requires a better understanding of the natural history of the disease. Here, we set up this clinical cohort study for evaluating patients with HSP from clinical, MRI, cognitive, genetic and biochemical perspectives, to increase our understanding of the natural history of these diseases and facilitate future diagnoses and therapy.

\section{OBJECTIVES}

\section{Primary objectives}

- Obtain genetic-clinical spectrum characteristics in patients with HSP.

- Record changes from baseline of Spastic Paraplegia Rating Scale (SPRS) total score across 3 years.

- Characterise mechanistic contributions of HSP subtypes with different cellular pathways to aid in estimating likely disease severity.

\section{Secondary objectives}

- Record changes in MRI of brain and spinal cord (C1T10) over 3 years.

- Record changes from baseline in cognitive competence score over 3 years.

- Record plasma levels of neurofilament-light peptide (NF-L), tau protein and myelin basic protein (MBP) at stages including presymptomatic HSP, phenoconversion and HSP.

\section{METHOD AND ANALYSIS}

\section{Study design}

This is a case-control parallel cohort study. We will recruit patients with HSP who have been previously diagnosed in our centre (about 300 patients) and some patients currently being diagnosed in our centre. At present, we diagnose 5-8patients/month, so it is feasible to recruit 200 HSP patients over the 12-month period from March 2021 to March 2022. After the recruitment period and obtaining the signed informed consent from all the participants, a $10.0 \mathrm{~mL}$ venous blood sample will be collected from each participant. Six aspects, including clinical signs, genetic spectrum, cognitive competence, MRI features, potential biochemical indicators and nerve electrophysiological factors will be collected. Evaluations would be conducted according to the testing schedule shown in table 1 .

\section{Participants}

Inclusion criteria

Candidates meeting one of the following criteria will be enrolled:

- Candidate with progressive spastic gait disturbance.

- Bilateral lower limb spasticity, hyperreflexia and extensor plantar responses.

- Candidate with HSP gene diagnosis with or without symptoms.

\section{Exclusion criteria}

Candidates with any of the following disqualifying criteria will be excluded:

- Candidate is unable to provide consent.

- Candidate has structural abnormalities or severe lesions in the brain and/or spinal cord.

- Candidate with disability caused by severe lower extremity trauma or has undergone major surgery resulting in lower extremity disability.

- Candidate with spinocerebellar ataxias (SCAs) that may cause corticospinal tract impairment.

- Other diseases cause spastic paraplegia, including tropical spastic paraplegia, subacute combined degeneration and vitamin $\mathrm{E}$ deficiency.

\section{Screening}

Some other diseases, such as vitamin B12 deficiency, vitamin E deficiency, syphilis, AIDS and brain or spinal cord injury, may also present with spastic paraplegia symptoms. In addition, genetic disorders such as SCAs may also mimic HSP in the clinic. ${ }^{13}$ Moreover, as whole exome sequencing (WES) often performs poorly with polynucleotide duplicates, this technique is largely unsuitable for screening SCAs, many of which are associated with polynucleotide repeat sequences. Therefore, we set up a screening system to remove such candidates from the pool (figure 1).

First, plasma homocysteine, vitamin B12, folic acid and vitamin $\mathrm{E}$ will be measured in the clinical laboratory of the First Affiliated Hospital of Fujian Medical University to exclude subacute combined degeneration and vitamin $\mathrm{E}$ deficiency. An HIV antibody test and treponema pallidum gelatin granule agglutination test will also be performed in this clinical laboratory to exclude HIV and treponema pallidum infection. An anti-HTLV-1/2 enzyme-linked 
Table 1 Study schedule

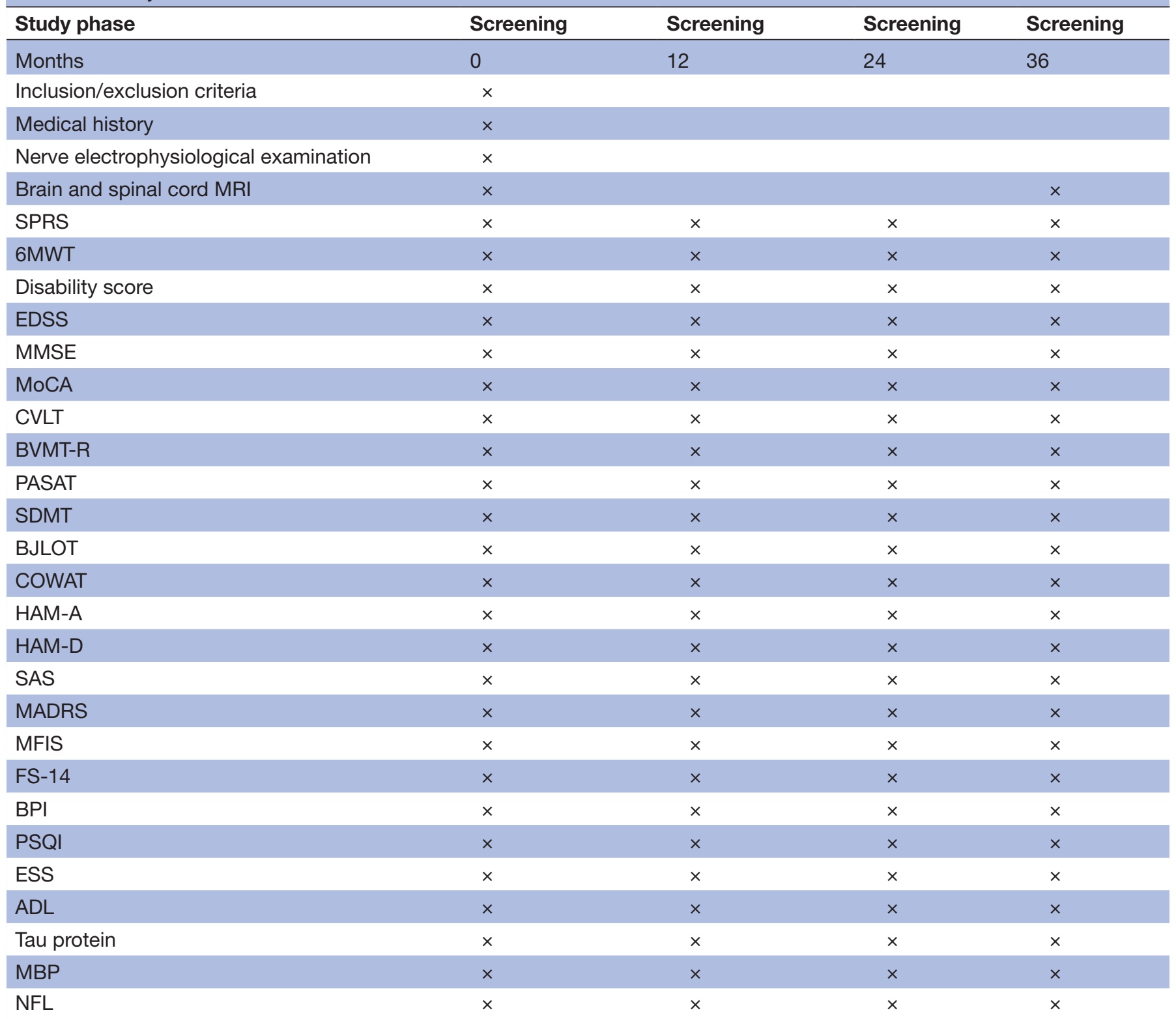

ADL, Activity of Daily Living; BJLOT, Benton Judgement of Line Orientation test; BPI, Brief Pain Inventory; BVMT-R, Brief Visuospatial Memeory Test-Revised; COWAT, Controlled Oral Word Association Test; CVLT, California Verbal Learning Test; Disability score, SPATAXEUROSPA disability stage; EDSS, Expanded Disability Status Scale; ESS, The Epworth Sleeping Scale; FS-14, Fatigue Scale-14; HAM-A, Hamilton Anxiety Scale; HAM-D, Hamilton Depression Scale; MADRS, Montgomery-Åsberg Depression Rating Scale; MBP, myelin basic protein; MFIS, Modified Fatigue Impact Scale; MMSE, Mini Mental State Examination; MoCA, Montreal Cognitive Assessment Beijing Version; 6MWT, 6-Minute Walk Test; NFL, neurofilament protein-light; PASAT, Paced Auditory Serial Addition Test; PSQI, Pittsburgh Sleep Quality Index; SAS, Self-Rating Anxiety Scale; SDMT, Symbol Digit Modalities Test; SPRS, Spastic Paraplegia Rating Scale.

immunosorbent assay (Wantai, Beijing, China) will be used for screening Human T-lymphotropic virus (HTLV) infection to exclude tropical spastic paraplegia. MRI of the cerebral, cervical and thoracic medullary will be performed to exclude structural abnormalities or severe lesions that may cause spastic paraplegia. Agarose gel electrophoresis will be used to detect multinucleotide duplication number of some SCA genes to exclude SCA1, SCA2, SCA3, SCA6, SCA7, SCA8, SCA10, SCA12, SCA17, dentatorubral-pallidoluysian atrophy and Friedreich's ataxia. If the fragment length is abnormal, Sanger sequencing will be further performed to determine the number of repetitions. This procedure is shown in figure 1, and the primer is shown in online supplemental table S1.

\section{Recruitment}

Potential participants will be screened and those eligible will be approached by members of the Neurology team. Eligible triplets or whole families will be provided with the participant information sheet, invited to participate, and given the opportunity to ask questions. Written consent will be obtained prior to inclusion in the cohort. 


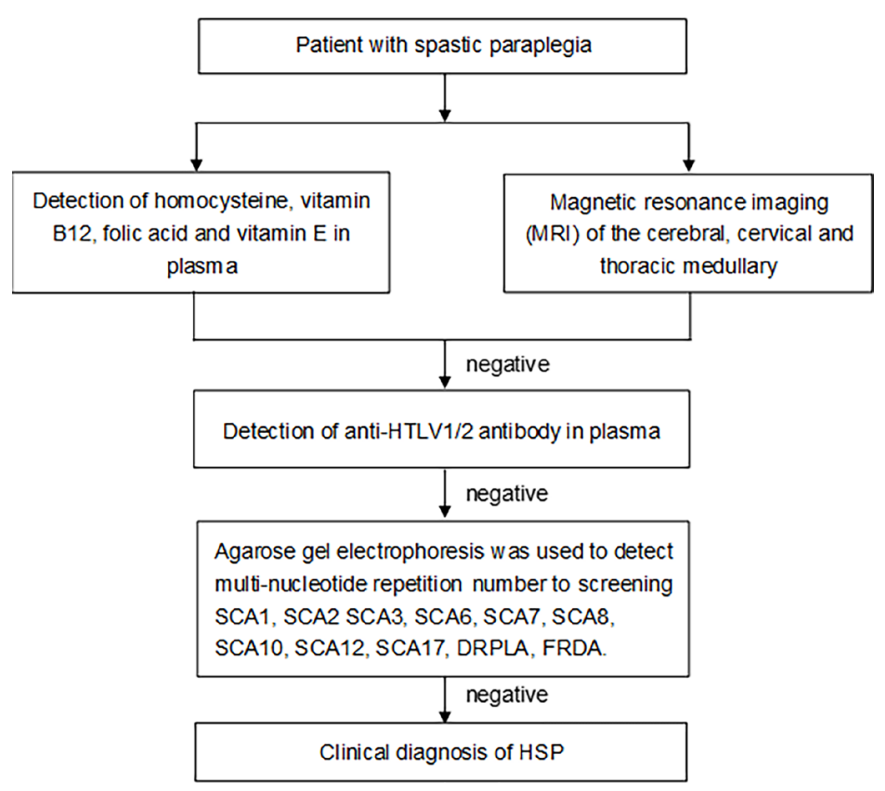

Figure 1 Clinical diagnosis procedure of HSP. DRPLA, dentatorubral-pallidoluysian atrophy; FRDA, Friedreich's ataxia; HSP, hereditary spastic paraplegia; SCA, spinocerebellar ataxia.

Assessment will begin after the informed consent has been signed.

\section{Study assessment}

Clinical feature collection

To quantify clinical severity and screen for complex symptoms, the neurological examination will be performed in the Neurological Evaluation Room of the First Affiliated Hospital of Fujian Medical University. We will use a standardised inventory to collect clinical features. Neurological examinations include assessment of mental status, level of consciousness, sensory function, motor function, cranial nerve function and reflexes. ${ }^{14}$ SPRS scores will be determined and assessed for every participant to quantify the severity of the disease. ${ }^{1516}$ The 6-Minute Walk Test will be performed, and if the participant is able to walk independently with or without assistance, a video of the test will be recorded. These tests will be performed at the times shown in the Schedule of Procedures, and all investigators will be trained in the administration of these tests.

We will also collect data regarding disease progression time points for each patient. Specifically, time of initial visit will be defined as the time of onset to the time a patient first visits a doctor because of abnormal gait. Crutch dependence onset time (if crutch dependence begins before or during the study) will be defined as the time from onset of abnormal gait to crutch dependence. Wheelchair dependence onset time (if wheelchair dependence begins before or during the study) will be defined as the time from onset of abnormal gait to wheelchair dependence.
Biochemical detection

The $10.0 \mathrm{~mL}$ venous blood samples collected at the First Affiliated Hospital of Fujian Medical University will be sent to the Medical University's Institute of Neurology within an hour. There, the blood will be centrifuged for $5 \mathrm{~min}$ at $3800 \mathrm{rpm}$, where a quality controller will observe whether the sample is clear and contains no precipitation, no serious hemolysis, etc. If the quality is not good, the blood sample will be discarded and collected again. Plasma will be dispensed into $1.5 \mathrm{~mL}$ centrifuge tubes with a total of 8 tubes of $200 \mu \mathrm{L}$ each tube, then the tubes will be stored in a refrigerator at $-80^{\circ} \mathrm{C}$. Repeated freezethaw cycles will be avoided. Samples will be stored for no more than 2 years before the start of measurements.

NF-L, MBP, tau protein and P-tau protein will be used as biomarkers of neurodegeneration to assess disease severity. ${ }^{17-19}$ NF-L will be measured in duplicate with the single molecule array (Simoa) NF-Light Advantage Kit (Quanterix, Billerica) on a Simoa HD-1 Analyzer instrument, according to the manufacturer's instructions (Simoa Homebrew Assay Development Guide). Similarly, P-tau protein will detect by Simoa Neurology 3-Plex A Kit from Quanterix and P-tau protein will be tested using Simoa P-tau-231 Advantage Kit (Quanterix). In addition, ELISA produced by R\&D Systems (Minneapolis; Catalog Number: DY4228-05) was used to detect MBP in duplicate. Samples for MBP will be measured three times; if a coefficient of variation greater than $10 \%$ compared with the mean value is obtained, the sample will be remeasured. All measurements will be performed by certified laboratory technicians.

\section{Gene detection}

To determine the genetic diagnosis, genomic DNA will be extracted from the peripheral blood of study participants using Qiagen kits (Hilden, Germany). We will use multiplex ligation-dependent probe amplification (MLPA) combined with WES to analyse genomic DNA. MLPA testing will be used to detect large deletions or copy number variations in commonly known pathogenic genes, followed by WES employed to screen for putative HSP disease-causing genes in all probands. The SALSA MLPA probe mixes (MRC-Holland; Amsterdam, Netherlands), including P165-C2, P211-B4, P306-B1 and P213-B2, will be used for the MLPA tests specifically. The detection range includes all exons of genes that are prone to insertions or deletions, including ATL1, SPAST, REEP1, SPG7, NIPA1 and SPG11 genes. Data will be collected and analysed by Genemapper V.3.0 (ThermoFisher; Waltham) and Coffalyser software (MRC-Holland), respectively, following standard protocols and data analysis pipelines. ${ }^{20}$

The QXT enzymatic protocol for adaptor tagging (Agilent Technologies; Santa Clara) will be used to fragment DNA, and the fragments will be purified by magnetic beads using the Agilent SureSelect Human All Exon $50 \mathrm{MB}$ (V.6) platform. Targeted regions will be captured with whole-exome oligonucleotides and amplified to prepare libraries. Next, paired-end reads will 
be generated from the libraries using the HiSeq 3000 sequencing platform. The reads will then be aligned to the human genome build $38^{21}$ using Burrows-Wheeler Aligner-MEM software. We will then analyse using the Genome Analysis Toolkit and annotate using ANNOVAR. ${ }^{22} 23$

Variant frequency will be filtered using three databases with a $<0.1 \%$ inclusion criterion (Exome Sequencing Project, Exome Aggregation Consortium database, China Metabolic Analytics Project and the Genome Aggregation Database). SIFT, PolyPhen-2 and MutationTaster will be used to predict the pathogenicity of genetic variants. The American College of Medical Genetics and Genomics criteria will be used to classify variants into pathogenic, likely pathogenic and variants of uncertain significance. And putative pathogenic variants will also be compared with the Human Gene Mutation Database. ${ }^{24} 25$

MRI data acquisition

MRI acquisition will be performed at 0 and 36 months (table 1). All subjects will be examined with a 3T MAGNETOM Skyra MRI Scanner (Siemens; Munich, Germany) equipped with a 20-channel head-neck coil and a 24-channel spine-array coil. Cervical and thoracic spinal cord images will be obtained using the following spin-echo sequences: 3D T2-weighted turbo spin-echo sequence (Sampling Perfection with Applicationoptimised Contrast using different flip angle Evolutions, SPACE); 3D-T1-weighted turbo spin-echo sequence (magnetisation prepared rapid acquisition gradient echo,
MPRAGE) in the sagittal plane; and three axial conventional sequences (2D T2* MEDIC (Multiple Echo Date Image Combination), 2D T2-PD and 2D T2). ${ }^{26}$ Spinal diffusion tensor imaging (DTI) T1 mapping and RESTBOLD (blood oxygen-level dependent) images were concurrently acquired. Brain images were also obtained including 3D-T1, 2D-T2, fluid attenuation inversion recovery, Susceptibility weighted imaging(SWI), DTI and REST-BOLD images. The parameters of the main MRI sequences are listed in table 2.

To obtain the volume of individual grey matter (GM, sum of voxels classified as GM), white matter (WM, sum of voxels classified as WM) and cerebrospinal fluid (CSF), 3D T1 images were examined and processed using the Computational Anatomy Toolbox V.12 (CAT V.12, http://www.neuro.uni-jena.de/cat/), an SPM V.12 add-on (Statistical Parametric Mapping V.12, http:/ / www. fil.ion.ucl.ac.uk/spm) running under MATLAB (R2013b, the MathWorks; Natick) as previously described. ${ }^{27-30}$ Briefly, structural MRI are segmented into GM, WM and CSF following the standard segmentation model and then spatially registered to the Montreal Neurological Institute (MNI) template using the affine registration algorithm. Afterwards, the GM and WM concentration images are non-linearly warped using the Diffeomorphic Anatomical Registration Through Exponentiated Lie Algebra algorithm and resampled to a voxel size of $1.5 \times 1.5 \times 1.5 \mathrm{~mm}$. Finally, the resulting images are modulated using the

Table 2 Parameters for the MRI sequences

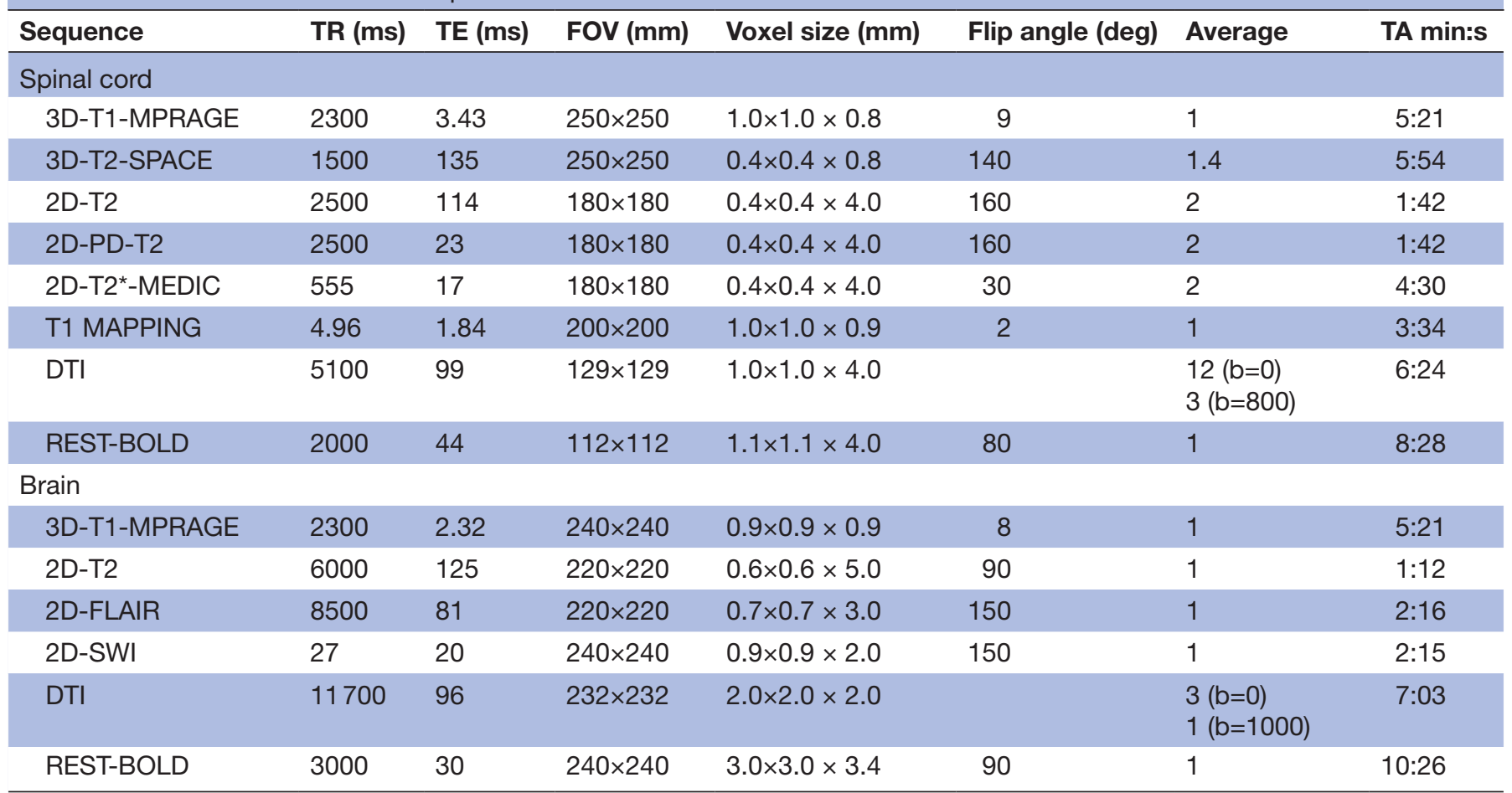

BOLD, blood oxygen-level dependent; DTI, diffusion tensor imaging; FLAIR, fluid attenuation inversion recovery; FOV, field of view,3D-T1MPRAGE, three-dimensional magnetisation prepared rapid acquisition gradient echo; 3D T2- SPACE, three-dimensional sampling perfection with application-optimised contrast by using different flip angle Evolutions; SWI, susceptibility weighted imaging; TA, acquisition time; TE, echo time; TR, repetition time. 
Jacobian determinant approach to compare the relative volume adjusted for individual brain size. Additionally, the total intracranial volume is defined as the sum of all voxels classified as grey or WM or CSF.

Voxel-wise tract-based spatial statistical analysis will be carried out using the default FSL (FMRIB Software Library; where FMRIB=Functional MRI of the Brain) pipeline for brain DTI data. ${ }^{31}{ }^{32}$ Brief steps are described as follows: (1) alignment of each fractional anisotropy (FA) image to every other one, (2) identification of the 'most representative image', (3) affine alignment of the identified target image into the MNI standard space, (4) transformation of every subject's image into standard MNI space by combining the non-linear transforms into the target image with the affine transformation from that target into MNI space, (5) creation of a representative mean FA 'skeleton'. Finally, the maximum value of each subject's FA data (basically, a vector) will then be projected onto this skeleton to be used as input for the voxel-wise statistical analysis.

The Spinal Cord Toolbox (V.4.01) ${ }^{33}$ will be used to segment and extract the morphological metrics of the spinal cord across vertebral level. ${ }^{33-36}$ To obtain spinal cord cross-sectional area and sectional diameters, the main steps are: (1) segmentation of spinal cord. For each participant, the spinal cord images are automatically segmented using the standard model known as PropSeg, with manual correction applied as necessary, (2) identification of vertebral levels. After the posterior tip of the C2-3 disc is identified programmatically, the C2-3 reference points are also set automatically. Results are checked in the quality control report, (3) template registration. The structural images are first straightened to match the orientation of the default template. Local deformations are then performed using affine transformations constrained in the axial plane, (4) extraction of relevant metrics. Once template registration has been completed, relevant metrics are automatically quantified programmatically.

Spinal DTI data are preprocessed, which includes segmenting. Motion correction is then performed, and DTI metrics are estimated. After this, the PAM50 template will be mapped onto DTI data along with crossmodal registration of the anatomical image to the mean diffusion-weighted image. Finally, the DTI metrics of the spinal cord will be extracted using maximum-likelihood with default parameters.

\section{Cognitive assessment}

We will perform neuropsychological evaluations of the participants over the contiguous 3-year period of the study at 1-year intervals. Age, handedness and education of the participants will also be recorded.

Our neuropsychological assessment may consist of two parts. The obligatory part is a general assessment of cognitive function, comprised of the Mini-mental State Examination and the Montreal Cognitive Assessment. The second part is five cognitive domains as follow:
Symbol Digit Modalities Test (SDMT) will be used to assess visual processing speed and working memory. ${ }^{37}$ Participants are expected to write the number corresponding with a specific symbol. Then, $90 \mathrm{~s}$ are allowed for each trial. Performance is assessed based on the number of correct responses.

California Verbal Learning Test (CVLT)-II will be used to test auditory and verbal episodic memory. ${ }^{38}$ The CVLT-II list contains 16 words that each belong to one of four categories (vegetables, animals, ways of travelling and or furniture). Assessment of episodic memory will be based on the scores achieved from five trials, and include free and cued trials of short and long delays, inserting and repeating numbers and recognition.

Brief Visuospatial Memory Test - Revised (BVMT-R) will be used to evaluate visual and spatial episodic memory. ${ }^{39}$ BVMT-R requires participants to remember an array of abstract geometric figures $(2 \times 3$ array $)$. with three learning trials of $10 \mathrm{~s}$ each. After the array is removed, patients will be required to draw the array, with the correct figures in the correct positions. Assessment is based off of the acquisition scores from three trials.

Benton Judgement of Line Orientation test (BJLOT) will be used to test spatial processing. ${ }^{40}$ The test requires patients to match 30 items consisting of different pairs of angled lines to display cards. The two item forms, $\mathrm{H}$ and $\mathrm{V}$, present the same items but in different order. The patient's score will depend on the number of items in which judgments for both lines are correct.

Controlled Oral Word Association Test (COWAT) will be used to evaluate semantic fluency. ${ }^{41}$ COWAT for English speakers asks participants to speak only words beginning with a designated letter, such as 'F', for $1 \mathrm{~min}$. In order to adapt COWAT to Chinese-speaking patients, we replaced the letter with a word. Participants should speak idioms beginning with 'ren' (people), 'ri' (day), and 'shi' (to be). The score is the number of correct idioms spoken in $1 \mathrm{~min}$.

Paced Auditory Serial Addition Test (PASAT) is a measure of sustained attention and information processing speed. ${ }^{42}$ Our use of PASAT requires subjects to listen to an audio recording of a voice stating 61 onedigit numbers; two versions of the PASAT administer the numbers at $3 \mathrm{~s}$ and $2 \mathrm{~s}$ intervals. The subject is then asked to add the last number heard to the previous number. The total score is the number of correct answers in each trial (range: $0-60$ ).

The above neuropsychological assessments have previously been validated in the general Chinese population. ${ }^{43} 44$ Specifically, the Chinese version of MATRICS Consensus Cognitive Battery ${ }^{45}$ which has been well validated in China, ${ }^{46}$ was used to adapt the PASAT, SDMT and BVMT-R. The Chinese version of CVLT-II has shown good reliability and validity in a previous study, ${ }^{47}$ and the BJLOT test, which consists of pairs of angled lines, shows no significant influence based on culture or language differences. ${ }^{48}$ The COWAT was modified due to the linguistic differences between Chinese logograms and languages that employ an alphabet ${ }^{44}$. 
Neuropsychological, sleep and activities of daily living also will be monitored with a battery questionnaires comprised of the Hamilton Anxiety Rating Scale, the Hamilton Depression Rating Scale (HAM-D) ${ }^{49}$ the SelfRating Anxiety Scale, the Montgomery-Åsberg Depression Rating Scale, the Modified Fatigue Impact Scale, the Fatigue Scale-14, the Brief Pain Inventory, the Pittsburgh Sleep Quality Index, the Epworth Sleepiness Scale and the Activity of Daily Living Scale.

\section{Nerve electrophysiological examination}

A nerve conduction study (NCS) and somatosensory evoked potential (SSEP) will be performed using KEYPOINT WORKSTATION. The skin temperature of all subjects will be maintained at or above $32^{\circ} \mathrm{C}-34^{\circ} \mathrm{C}$. The protocols of the NCSs include sensory (median, ulnar, radial, superficial peroneal, and sural) and motor (median, ulnar, common peroneal, and tibial) nerves on both sides. Recordings will be performed with standard settings: $0.2 \mathrm{~ms}$ stimulus duration, a $1 \mathrm{~Hz}$ stimulus frequency, $20 \mathrm{~Hz}-3 \mathrm{kHz}$ frequency bandwidth, and a sensitivity of $20 \mu \mathrm{V} /$ division for sensory, while $0.1 \mathrm{~ms}$ stimulus duration, a $1 \mathrm{~Hz}$ stimulus frequency, $20 \mathrm{~Hz}-10 \mathrm{kHz}$ frequency bandwidth, and a sensitivity of $5 \mathrm{mV} /$ division will be used for motor. We will record the amplitudes for all nerves, as measured from the main negative to the subsequent positive peak ('peak-to-peak amplitude').

The H-reflex of the tibial nerve will be measured as the stimulation gradually increases. H-reflex latency will be expressed in milliseconds, while the ratio of the maximum H-reflex to that of the maximum compound muscle action potential of the soleus muscle will be used to express amplitude. A total of 20 consecutive supramaximal stimuli will be used to study the $\mathrm{F}$ response of the median nerve to routine stimulation (wrist) and recording techniques (abductor pollicis brevis). We will also analyse F-wave persistence and conduction velocity using a sensitivity of $200 \mu \mathrm{V} /$ division and filter setting of $20 \mathrm{~Hz}-10 \mathrm{kHz}$. SSEP of the tibial will be measured three times. The stimulus, a single square wave of $200 \mu$ s, will be delivered to the tibial nerve adjacent and posterior to the medial malleolus at a frequency of $3.1 \mathrm{~Hz}$. After the patient is comfortable and relaxed, the stimulus will be applied with a maximal intensity limited to $20.0 \mathrm{~mA}$. The intensity of the stimulus will be adjusted in order to elicit a slight but visible twitch at the hallux of the ipsilateral foot. The recording bandwidth will be $30 \mathrm{~Hz}$ to $3 \mathrm{kHz}$. Data collection will be terminated once an average of 500 trials have been performed for each channel. The results of the NCS and SSEP experiments will be compared with the reference ranges in our laboratory, which were determined from age-matched and sex-matched healthy adult volunteers using the same protocols.

\section{Patient and public involvement}

The research question and design of this study were formulated without input from patients and the public.

\section{Statistical plan}

Analysis and generation of plots and visualisations will be performed using SPSS software (V25; IBM; Armonk). Descriptive statistics will be used for analysing the data distribution. Missing data will be addressed by deletion or through use of the last observation carried forward, determined on a case-specific basis. Single variable analysis will be implemented to analyse the correlations between exposures, possible confounding conditions and outcomes. The Kolmogorov-Smirnov test or Shapiro-Wilk test will be used to assess the normality of the variables. Continuous variables will be presented as the median (range). For normally distributed variables, group differences will be assessed by independent two-tailed Student's t test or analysis of variance. For variables that are not normally distributed, the Mann-Whitney U test or Kruskal-Wall test will be used, as appropriate, or the data will be log transformed for parametric testing (Spastic Paraplegia(SPG) type, age, sex, etc). Multiple linear regression analysis will be applied to track the interaction with SPRS score and cognitive correlation to MRI, age of onset, sex and biochemical data. Categorical variables will be compared for the groups using the $\mathrm{X}^{2}$ test (Fisher's exact test when the expected value is $<5$ ). Values of $\mathrm{p}<0.05$ will be considered statistically significant. Bonferroni correction will be applied to adjust for multiple comparisons.

\section{DATA MANAGEMENT AND ELECTRONIC SYSTEM}

The Institute of Neurology of First Affiliated Hospital will maintain a data management plan specifying all relevant aspects of data processing for the study (including data validation, cleaning, correcting, releasing).

The processes and the dictionaries used for coding will be specified in a medical coding plan. Internationally recognised and accepted dictionaries will be used for all data coding (eg, AEs, baseline findings, medication, medical/surgical history/ophthalmic history).

The case report form data for this study will be collected with an ecosystem provided by Yidu Cloud (Beijing, China). Users will be trained before they are granted access to the system.

\section{ETHICS AND DISSEMINATION}

This study was granted ethics committee approval by the First Affiliated Hospital of Fujian Medical University (MRCTA, ECFAH of FMU (2019)194) in 2019. For all research activities, informed consent will be obtained from the participants or from the parents or legal guardians of participants under the age of 18 . For the consideration of face-to-face data collection, researchers will follow their institutional and/or government guidelines to protect themselves and participants from COVID-19 infection. Data will be stored on the Yidu cloud server, and only research team members will have access to the data during the study. Traditional dissemination methods will include publishing open-access information in 
peer-reviewed journals and presenting research results at national and international conferences.

\section{Author affiliations}

${ }^{1}$ Department of Neurology and Institute of Neurology of The First Affiliated Hospital, Institute of Neuroscience, and Fujian Key Laboratory of Molecular Neurology, Fujian Medical University, Fuzhou, Fujian, China

${ }^{2}$ Department of Radiology of The First Affiliated Hospital, Fujian Medical University, Fuzhou, Fujian, China

${ }^{3}$ Department of Medical Imaging Technology, College of Medical Technology and Engineering, Fujian Medical University, Fuzhou, Fujian, China

${ }^{4}$ Department of Nuclear Medicine of The First Affiliated Hospital, Fujian Medical University, Fuzhou, Fujian, China

Contributors W-JC, YF, NW, YL conceptualised the study and participated in initial study design, with assistance from Y-SQ, Y-HZ, R-YY, Z-XY, JB, M-WW, Y-JC, XL and $X-H L$ drafted the manuscript. $Y$-SQ prepared visual content and coordinated manuscript revisions. W-JC, YF, NW, YL obtained the research funding. All other authors (Y-KC, YL, S-BY and J-YJ) were involved in the writing or review of the manuscript and approved the final version.

Funding This work was supported by the grants 82025012 (W-JC), U1905210 (W-JC), U2005201 (NW), 81771230 (W-JC), 81771279 (YF) and 81801130 (XL) from the National Natural Science Foundation of China, the Natural Science Foundation of Fujian Province 2019J02010 (W-JC) and 2019J05076 (XL), the Joint Funds for the Innovation of Science and Technology of Fujian Province 2017Y9094 (W-JC) and 2018Y9082 (NW), the Key Clinical Specialty Discipline Construction Program of Fujian (NW).

\section{Competing interests None declared.}

Patient consent for publication Consent obtained directly from patient(s)

Provenance and peer review Not commissioned; externally peer reviewed.

Supplemental material This content has been supplied by the author(s). It has not been vetted by BMJ Publishing Group Limited (BMJ) and may not have been peer-reviewed. Any opinions or recommendations discussed are solely those of the author(s) and are not endorsed by BMJ. BMJ disclaims all liability and responsibility arising from any reliance placed on the content. Where the content includes any translated material, BMJ does not warrant the accuracy and reliability of the translations (including but not limited to local regulations, clinical guidelines, terminology, drug names and drug dosages), and is not responsible for any error and/or omissions arising from translation and adaptation or otherwise.

Open access This is an open access article distributed in accordance with the Creative Commons Attribution Non Commercial (CC BY-NC 4.0) license, which permits others to distribute, remix, adapt, build upon this work non-commercially, and license their derivative works on different terms, provided the original work is properly cited, appropriate credit is given, any changes made indicated, and the use is non-commercial. See: http://creativecommons.org/licenses/by-nc/4.0/.

\section{ORCID iDs}

Ying Fu http://orcid.org/0000-0002-1590-3945

Wan-Jin Chen http://orcid.org/0000-0002-9472-8494

\section{REFERENCES}

1 Novarino G, Fenstermaker AG, Zaki MS, et al. Exome sequencing links corticospinal motor neuron disease to common neurodegenerative disorders. Science 2014;343:506-11.

2 Shribman S, Reid E, Crosby AH, et al. Hereditary spastic paraplegia: from diagnosis to emerging therapeutic approaches. Lancet Neurol 2019;18:1136-46.

3 Ruano L, Melo C, Silva MC, et al. The global epidemiology of hereditary ataxia and spastic paraplegia: a systematic review of prevalence studies. Neuroepidemiology 2014;42:174-83.

4 Schüle R, Schöls L. Genetics of hereditary spastic paraplegias. Semin Neurol 2011;31:484-93.

5 Dong E-L, Wang C, Wu S, et al. Clinical spectrum and genetic landscape for hereditary spastic paraplegias in China. Mol Neurodegener 2018;13:36.

6 Vaz FM, McDermott JH, Alders M, et al. Mutations in PCYT2 disrupt etherlipid biosynthesis and cause a complex hereditary spastic paraplegia. Brain 2019;142:3382-97.
7 Harding AE. Classification of the hereditary ataxias and paraplegias. Lancet 1983;1:1151-5.

8 Harding AE. Hereditary spastic paraplegias. Semin Neurol 1993;13:333-6.

9 Kara E, Tucci A, Manzoni C, et al. Genetic and phenotypic characterization of complex hereditary spastic paraplegia. Brain 2016:139:1904-18.

10 Schüle R, Wiethoff S, Martus P, et al. Hereditary spastic paraplegia: Clinicogenetic lessons from 608 patients. Ann Neurol 2016;79:646-58.

11 Fink JK. Hereditary spastic paraplegia: clinico-pathologic features and emerging molecular mechanisms. Acta Neuropathol 2013;126:307-28.

12 Blackstone C. Cellular pathways of hereditary spastic paraplegia. Annu Rev Neurosci 2012;35:25-47.

13 Wang Y-guang, Du J, Wang J-ling, et al. Six cases of SCA3/MJD patients that mimic hereditary spastic paraplegia in clinic. $J$ Neurol Sci 2009;285:121-4.

14 Diniz de Lima F, Faber I, Servelhere KR, et al. Randomized trial of botulinum toxin type $A$ in hereditary spastic paraplegia - the SPASTOX trial. Mov Disord 2021;36:1654-63.

15 Schüle R, Holland-Letz T, Klimpe S, et al. The spastic paraplegia rating scale (SPRS): a reliable and valid measure of disease severity. Neurology 2006;67:430-4.

16 Chrestian N, Dupré N, Gan-Or Z, et al. Clinical and genetic study of hereditary spastic paraplegia in Canada. Neurol Genet 2017;3:e122.

17 Brureau A, Blanchard-Bregeon V, Pech C, et al. NF-L in cerebrospinal fluid and serum is a biomarker of neuronal damage in an inducible mouse model of neurodegeneration. Neurobiol Dis 2017;104:73-84.

18 Vassall KA, Bamm V, Harauz G. MyelStones: the executive roles of myelin basic protein in myelin assembly and destabilization in multiple sclerosis. Biochem J 2015;472:17-32.

19 Avila J, Lucas JJ, Perez M, et al. Role of tau protein in both physiological and pathological conditions. Physiol Rev 2004;84:361-84.

20 Zhao M, Chen Y-J, Wang M-W, et al. Genetic and clinical profile of Chinese patients with autosomal dominant spastic paraplegia. Mol Diagn Ther 2019;23:781-9.

$21 \mathrm{Li} \mathrm{H}$, Durbin R. Fast and accurate short read alignment with BurrowsWheeler transform. Bioinformatics 2009;25:1754-60.

22 McKenna A, Hanna M, Banks E, et al. The genome analysis toolkit: a MapReduce framework for analyzing next-generation DNA sequencing data. Genome Res 2010;20:1297-303.

23 Wang K, Li M, Hakonarson H. ANNOVAR: functional annotation of genetic variants from high-throughput sequencing data. Nucleic Acids Res 2010;38:e164.

24 Caspar SM, Dubacher N, Kopps AM, et al. Clinical sequencing: from raw data to diagnosis with lifetime value. Clin Genet 2018;93:508-19.

25 Stenson PD, Ball EV, Mort M, et al. Human gene mutation database (HGMD): 2003 update. Hum Mutat 2003;21:577-81.

26 Ning Wang YL, Ye Z, Lin Y, et al. Cross sign T2 hyperintensities in atrophic spinal cord of hereditary spastic paraplegia type 5 . Available: https://www.researchsquare.com/article/rs-97876/v1

27 Tavares V, Prata D, Ferreira HA. Comparing SPM12 and CAT12 segmentation pipelines: a brain tissue volume-based age and Alzheimer's disease study. J Neurosci Methods 2019;334:108565.

28 Coppola G, Petolicchio B, Di Renzo A, et al. Cerebral gray matter volume in patients with chronic migraine: correlations with clinical features. J Headache Pain 2017;18:115.

29 Zhao C, Zhu J, Liu X, et al. Structural and functional brain abnormalities in schizophrenia: a cross-sectional study at different stages of the disease. Prog Neuropsychopharmacol Biol Psychiatry 2018;83:27-32.

30 Spalthoff R, Gaser C, Nenadić I. Altered gyrification in schizophrenia and its relation to other morphometric markers. Schizophr Res 2018;202:195-202.

31 Mascalchi M, Salvadori E, Toschi N, et al. DTI-derived indexes of brain WM correlate with cognitive performance in vascular $\mathrm{MCl}$ and small-vessel disease. A TBSS study. Brain Imaging Behav 2019;13:594-602.

32 Castellano A, Papinutto N, Cadioli M, et al. Quantitative MRI of the spinal cord and brain in adrenomyeloneuropathy: in vivo assessment of structural changes. Brain 2016;139:1735-46.

33 De Leener B, Lévy S, Dupont SM, et al. Sct: spinal cord toolbox, an open-source software for processing spinal cord MRI data. Neuroimage 2017;145:24-43.

34 Dupont SM, De Leener B, Taso M, et al. Fully-integrated framework for the segmentation and registration of the spinal cord white and gray matter. Neuroimage 2017;150:358-72. 
35 Gros C, De Leener B, Badji A, et al. Automatic segmentation of the spinal cord and intramedullary multiple sclerosis lesions with convolutional neural networks. Neuroimage 2019;184:901-15.

36 Moccia M, Prados F, Filippi M, et al. Longitudinal spinal cord atrophy in multiple sclerosis using the generalized boundary shift integral. Ann Neurol 2019;86:704-13.

37 Benedict RH, DeLuca J, Phillips G, et al. Validity of the symbol digit modalities test as a cognition performance outcome measure for multiple sclerosis. Mult Scler 2017;23:721-33.

38 Elwood RW. The California verbal learning test: psychometric characteristics and clinical application. Neuropsychol Rev 1995:5:173-201.

39 Pliskin JI, DeDios Stern S, Resch ZJ, et al. Comparing the psychometric properties of eight embedded performance validity tests in the Rey auditory verbal learning test, Wechsler memory scale logical memory, and brief visuospatial memory test-revised recognition trials for detecting invalid neuropsychological test performance. Assessment 2021;28:1871-81.

40 Calamia M, Markon K, Denburg NL, et al. Developing a short form of Benton's judgment of line orientation test: an item response theory approach. Clin Neuropsychol 2011;25:670-84.

41 Sumerall SW, Timmons PL, James AL, et al. Expanded norms for the controlled oral word association test. J Clin Psychol 1997;53:517-21.
42 Tombaugh TN. A comprehensive review of the paced auditory serial addition test (PASAT). Arch Clin Neuropsychol 2006;21:53-76.

43 Bo Q, Mao Z, Li X, et al. Use of the MATRICS consensus cognitive battery (MCCB) to evaluate cognitive deficits in bipolar disorder: a systematic review and meta-analysis. PLoS One 2017; 12:e0176212.

44 Zhang N, Li YJ, Fu Y, et al. Cognitive impairment in Chinese neuromyelitis optica. Mult Scler 2015;21:1839-46.

45 Shi C, Kang L, Yao S, et al. What is the optimal neuropsychological test battery for schizophrenia in China? Schizophr Res 2019;208:317-23.

46 Shi C, Kang L, Yao S, et al. The MATRICS consensus cognitive battery (MCCB): Co-norming and standardization in China. Schizophr Res 2015;169:109-15.

47 Zhang Y, Zhu D, Zhang P, et al. Neural mechanisms of AVPR1A RS3-RS1 haplotypes that impact verbal learning and memory. Neuroimage 2020;222:117283.

48 Liao Z, Bu Y, Li M, et al. Remote ischemic conditioning improves cognition in patients with subcortical ischemic vascular dementia. BMC Neurol 2019;19:206.

49 Sun XY, Li YX, Yu CQ, et al. [Reliability and validity of depression scales of Chinese version: a systematic review]. Zhonghua Liu Xing Bing Xue Za Zhi 2017;38:110-6. 